- Palabras clave/ Didáctica proyectual, fenomenología, actos, protofenómeno arquitectónico.

$\Delta$ Keywords/ project didactics, phenomenology. acts, architectural proto-phenomenon.

$\Delta$ Recepción/ 1 noviembre 2017

$\Delta$ Aceptación/ 24 mayo 2018

\title{
Objetivo y objeto de estudio propio del arquitecto. Una distinción basal necesaria para el cultivo de una academia propiamente arquitectónica ${ }^{1}$
}

\section{Concerning the architect s objective} and his object of study. A necessary basal distinction for the cultivation of a properly architectonic academy ${ }^{1}$

\author{
Claudio Araneda \\ Arquitecto, Universidad del Bío-Bío, Chile. \\ Ph.D en Arquitectura, Architectural Association, \\ School of Architecture, Reino Unido. \\ Académico, Facultad de Arquitectura \\ Construcción y Diseño, Universidad del Bío-Bío, \\ Chile. \\ caraneda@ubiobio.cl
}

RESUMEN/ El presente artículo ofrece reflexiones actualizadas tanto respecto a las fuentes de conocimiento arquitectónico derivadas de un proyecto de investigación llevado a cabo en la Escuela de Arquitectura de la Universidad del Bío-Bío así como entorno a la posterior creación del curso Fundamentos de la Arquitectura, al que el proyecto contribuyó directamente y cuyo objetivo central es indagar sobre la fuente original de conocimiento arquitectural. Impulsado por la máxima fenomenológica que dicta que la generación de todo conocimiento presupone la existencia de un objeto de estudio de donde extraerlo, el artículo centra su atención en el primer ciclo de clases, basado en una aproximación fenoménica progresiva a los distintos reinos que componen nuestro mundo sensorial: reino mineral, vegetal, animal y 'humanal'. El presente trabajo presenta un análisis crítico a la tradición prevalente del aprendizaje en base al estudio de referentes, pone de manifiesto la incertidumbre epistemológica actual subyacente en la disciplina y ofrece líneas de investigación alternativas y complementarias. ABSTRACT/ This article provides updated reflections concerning both the sources of architectural knowledge arising from a research project conducted in the School of Architecture of the Universidad del Bío-Bío and the subsequent creation of the course on the Bases of Architecture, directly informed by the project and the main goal of which was to inquire on the original source of architectural knowledge. Promoted by the phenomenological core idea that dictates that the generation of all knowledge presumes the existence of a study object from where such knowledge can be extracted, the article focuses on the first class cycle based on a progressive phenomenology approach to the different kingdoms that make up our sensory world: the mineral, vegetal, animal and 'humanal' kingdoms. The work provides a critical analysis of the tradition prevalent in learning based on the study of models, reveals the current underlying epistemological uncertainty in the discipline, and offers optional and complementary lines of research.

\section{ANTECEDENTES EPISTEMOLÓGICOS BASALES.}

"No se busque nada detrás de los fenómenos: ellos mismos son la teoría" (Goethe 1993)
Hasta hace poco más de dos siglos, el conocimiento arquitectónico emanó casi exclusivamente de la tratadística arquitectónica, tradición inaugurada por el re-descubrimiento de los diez libros de Vitruvio y, desde entonces, actualizada a intervalos más o menos regulares por Alberti, Palladio, Laugier y, más recientemente, por el arquitecto Holandés Hans van der Laan (1983). Hacia mediados del siglo XVIII, sin embargo, la autoridad del tratado comienza a menguar de forma sostenida. Introduciendo un compendio actualizado de teoría arquitectural, Evers
(2006) confirma que el comienzo de la caída de la autoridad del canon tríadico vitruviano tuvo lugar alrededor de 1750, lo que no fue sino uno de los tantos efectos colaterales de una revolución epistemológica de mayor envergadura caracterizada por el advenimiento de la era de la razón o, si se prefiere, por la caída de la era de la fe, impulso que en los albores del siglo XIX -producto de la crisis de la ciencia física- derivó en la revolución fenomenológica que dio a luz a las llamadas ciencias de la vida.

El presente artículo se enmarca dentro de las actividades llevadas a cabo por el Grupo de Investigación en Didáctica Proyectual, perteneciente al Departamento de Diseño y Teoría de la Arquitectura de la Universidad del Bio-Bio, y se basa 
En arquitectura, este giro epistemológico significó el comienzo de la autoridad del referente histórico como fuente fidedigna de conocimiento arquitectónico y, con ello, el advenimiento del historicismo y del neoclasicismo². En este sentido, la crítica de Hillier (1996) sea probablemente la más articulada y fértil de las últimas décadas. Caracterizó la tradición tratadística como prescriptivamente fuerte pero analíticamente débil y, acto seguido, propuso una teoría propia que, basada en una fenomenología del trazado urbano, definió un antes y un después en la generación de conocimiento urbano, instalando el paradigma del análisis y diseño basados en evidencia ${ }^{3}$.

La radicalidad y vigencia de este giro epistémico en la búsqueda de conocimiento arquitectónico fue capturado con sorprendente honestidad por Pérez, Aravena y Quintanilla. Introduciendo un texto expresamente dirigido a estudiantes y profesores de arquitectura, escriben: "No tiene demasiado sentido preguntarse qué es la arquitectura, como si ella naciera con nosotros o, más exactamente, con nuestra pregunta. La arquitectura está alli en las obras que se conservan enteras o en ruinas, en proyectos, bocetos o escritos. No cabe inventarla, sino interpretarla y reconstruirla. Más sentido tiene, entonces, preguntarse por lo que la arquitectura ha sido"

(Pérez, Aravena y Quintanilla 1999: 31).

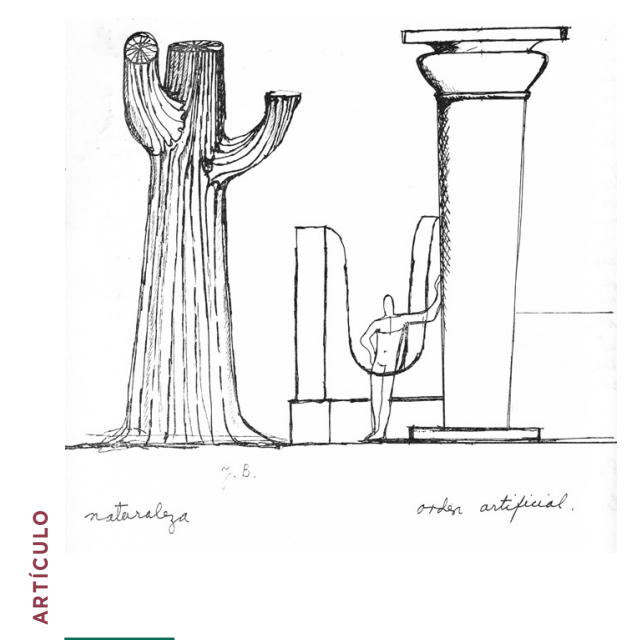

Dos sentencias se desprenden con claridad de este texto. Primero, no tiene sentido preguntarse qué es la arquitectura; y segundo, sí tiene sentido estudiar la obra construida, el fenómeno construido. Considerando que el cuerpo del texto lo constituyen una serie de ensayos sobre obras paradigmáticas de todos los tiempos, el mensaje no podría ser más claro: el conocimiento arquitectural subyace en las obras construidas; solo hace falta extraerlo. Las implicancias de esta cosmovisión arquitectónica son profundas. De entre ellas, probablemente la más grave para la formación de arquitectos sea el advenimiento de la idea fatalista, propia del fin de milenio, que afirma que todo está hecho y solo queda reinterpretar la historia construida, antigua o efecto reciente. Como efecto directo de lo anterior, tenemos la caída en descrédito de tareas propias del quehacer artístico como el cultivo disciplinado de la facultad de la imaginación $y$, con ello, de nociones connaturalmente artísticas como 'creatividad' u 'originalidad' (Piñón 2006: 64). Esto ha implicado la transformación efectiva de los estudios de arquitectura y urbanismo en una suerte de arqueología del universo construido. Dada la delicada naturaleza del argumento que aquí se hilvana, no está demás consignar aquí algo que podría parecer obvio: es un hecho irrefutable que se aprende mucho del estudio de los hechos construidos. Sin embargo, la aceptación tácita de la obra de arquitectura como fuente original de conocimiento arquitectural plantea paradojas irresueltas tanto por la academia como por la profesión. Concretamente -y esto es lo decisivo- deja sin respuesta la pregunta por el origen de las primeras manifestaciones arquitecturales sobre el planeta, así como también deja sin resolver la pregunta por las fuentes que informaron el primer tratado de arquitectura, el cual, dicho sea de paso, sigue vigente en lo sustancial.
Respecto a este último problema, Borchers fue terminante:

"Un acto propiamente Arquitectónico [...] no dispone de algo ya dado en la naturaleza. No está en tal situación el físico, pues el fenómeno se le presenta dado en la naturaleza [...] El Acto Arquitectónico propiamente tal, con todo de no dado en el mundo natural no es un invento [...] sino que es un Descubrimiento. Como tal aparece inusitado, pues antes de ocurrir no hay un indicio de observación posible y aparece como un efecto de una causa desconocida. La consecuencia ontológica de estos dos postulados reunidos es que: Un Acto Arquitectónico que provenga de otro Acto Arquitectónico ya realizado, es decir, una obra de Arquitectura ya hecha [...] es un Acto fallido, un error en el origen, en el ser mismo [...]" (Borchers 1965: 61).

Borchers (1965) afirma así que la aceptación de la obra construida como fuente original del saber arquitectural presupone el olvido sistemático de que la arquitectura, en tanto conquista humana, como todo arte, no pertenece al orden natural -al orden dado- sino que constituye un orden artificial. Luhmann (2000) haría referencia a un sistema social y, en tanto tal, de factura humana. Esta distinción entre orden natural y orden artificial (imagen 1) constituye la piedra fundacional sobre la que Hans van der Laan, referente bibliográfico fundamental de la obra teórica Borchiana, erigió su edificio teórico/práctico, cristalizado en su tratado y en la porción de obras de las que fue responsable. Tomada en serio, esta aseveración resulta alarmante, pues deja la profesión y la academia actual sin suelo firme donde pisar. En efecto, barre con dos de los supuestos más atesorados por los arquitectos de comienzo de siglo, a saber: que el conocimiento arquitectónico estaría incrustado, si no en la naturaleza, en las obras construidas. Esto abre un abismo epistemológico disciplinar. Y es
Imagen 1. Distinción entre orden natural y orden artificial segun Borchers. Al centro, un "objeto" representativo del "reino humanal", responsable de la creación e imposición del orden artificial sobre la faz de la tierra (fuente: Borchers 1975). 
aquí, al borde de este abismo, donde resuenan las preguntas disciplinares más urgentes que aún no tienen respuesta: ¿De dónde extrajeron su conocimiento los primeros arquitectos, responsables por las asombrosas y siempre enigmáticas primeras manifestaciones del orden arquitectural, si antes de ellas no había referente escrito o construido alguno de donde extraerlo? ¿Cuál fue el objeto a cuyo estudio se consagraron los protoarquitectos para dar a luz a las primeras manifestaciones del orden arquitectónico sobre el planeta? Aproximarnos a una respuesta actualizada a estas interrogantes constituye la razón de ser del curso Fundamentos de la Arquitectura, cuya génesis introducimos

ANTECEDENTES INSTITUCIONALES Y ACADÉMICOS. pedagógicas más significativa del Proceso de Bolonia fue el desplazamiento del énfasis casi exclusivo en los resultados del proceso de enseñanza/aprendizaje a uno puesto en el proceso mismo. La enseñanza de la arquitectura no fue indiferente a este cambio de paradigma. Si bien por razones de idiosincrasia, ésta siempre se ha basado en el aprendizaje a través de proyectos, a la hora de evaluar, el énfasis estuvo por largo tiempo puesto en el llamado 'proyecto final' (Schon 1987) ${ }^{4}$. Con Bolonia, se dio importancia a poner acento en los procesos de enseñanza/aprendizaje, lo que, de paso, instaló la utilización de portafolios como herramienta pedagógica fundamental (Seldin, 2010; Zubizarreta 2004). Ahora bien, en el entendido de que todo proceso tiene un principio y un fin, resulta evidente que igual atención debe prestarse al principio del proceso enseñanza/ aprendizaje o, lo que es lo mismo, al inicio del proceso didáctico proyectual. Y ocurre que el comienzo de este proceso depende exclusivamente del profesor a cargo.
Entonces, las preguntas clave van aquí dirigidas al profesor: ¿De dónde obtiene el conocimiento arquitectónico con el que gatilla, conduce y lleva a buen término su proceso de enseñanza? ¿Cuál es su fuente de conocimiento arquitectónico? ¿Cuál es su objeto de estudio primordial? O bien, ¿a dónde envía a sus alumnos a buscar el conocimiento arquitectónico con el que posteriormente informarán sus proyectos de arquitectura?

Con el objetivo de arrojar luz sobre este oscuro rincón del proceso didáctico/ proyectual, realizamos una convocatoria abierta al cuerpo docente del departamento de diseño y teoría de la arquitectura de la Universidad del Bío-Bío, a la cual acudió un total de diez docentes, todos profesores a cargo de talleres de proyecto ${ }^{5}$. Realizando focus groups quincenales y animados por la máxima fenomenológica que dicta que la generación de todo conocimiento presupone la distinción clara de un objeto de estudio del cual extraerlo, así como del establecimiento de un lazo empático y reverente con este (Seamon y Zajonc 1998), cada profesor dispuso de una sesión para dar respuesta a estas preguntas basales. Consultados expresamente por el objeto de estudio o fuente original del conocimiento arquitectónico, ninguno apuntó en la dirección del referente construido ${ }^{6}$ Sintetizando y condensando las respuestas en 7 frases clave, los participantes respondieron en los siguientes términos:

1. El habitar

2. El habitante o usuario

3. Las rutinas humanas cotidianas

4. Actividades del ser humano en el espacio

5. El acto entendido como actividad

humana en el espacio

6. Estudio del ser humano en cuerpo, alma y espiritu

7. La naturaleza humana entendida como un conjunto de sentidos
Frente a esto, dos hechos de común ocurrencia en nuestras escuelas siguen resultando inexplicables o, a lo menos, controversiales. Primero, que aún no haya acuerdo sobre si el proceso proyectual, a cualquier nivel, debe principiar o fundarse en un estudio del espacio (léase referentes) o de lo que en Chile ha venido a llamarse, con distintas acepciones, el "acto" (Borchers 1968, 1975; Cruz 2012; De la Cruz 2013, $2000)^{7}$, y que el comienzo del proceso didáctico proyectual sea improvisado o anecdótico. Segundo que de acuerdo a resultados aún no publicados del trabajo realizado en el curso "Teoría y Planificación Curricular" del Magíster en Didáctica Proyectual de la Universidad del Bío-Bío (a excepción del curso "Cultura del Cuerpo"8 dictado en la Escuela de Arquitectura de la Universidad Católica de Valparaíso), ningún curso troncal de malla curricular alguna en Chile se ocupe de forma sistemática y sostenida de aquello que declaramos de forma casi unánime como fuente original e inagotable de conocimiento arquitectónico. En efecto, el carácter eminentemente ejecutivo del taller de proyectos nunca ha provisto ni el espacio ni el tiempo adecuados para profundizar en un estudio de este tipo. Los cursos de historia y teoría tampoco lo han hecho, pues su razón de ser, al decir de Pérez, Aravena y Quintanilla (1999), es precisamente ponernos en contacto con los hechos construidos. A la luz de esta experiencia académica, la evidencia por ella proporcionada y en el contexto de un cambio de plan de estudios, se abre de forma expresa un nicho curricular cuyo objetivo no es otro que poner al estudiante de arquitectura en contacto con las preguntas epistémicas disciplinares fundamentales. Dictado en tres semestres en un lapso de tres años, el curso fue llamado "Fundamentos de la Arquitectura".

${ }^{4}$ Hay excepciones paradigmáticas tales como la Bauhaus, la Architectural Association, la Escuela de Arquitectura de la Universidad Católica de Valparaíso en Chile, y otras menos conocidas (Colomina s/f).

${ }^{5}$ El proyecto llevó por nombre "Ur_Lab", fue financiado por la Dirección de Investigación de la Universidad del Bío-Bío (DIUBB 111902) y desarrollado durante el año 2012. Su objetivo central fue el de formular la pregunta por el objeto de estudio propio del arquitecto, de modo de sentar bases epistemológicas para la apertura de un laboratorio para el estudio del 'habitante arquetípico.

${ }^{6}$ El mismo ejercicio, en formato de workshop, fue realizado por el Magister en Didáctica Proyectual de la Universidad del Bio-Bio con un grupo aproximado de 15 profesores de la Escuela de Arquitectura de la Universidad Nacional de Colombia, sede Medellín (2015), y con otro de aproximadamente 50 profesores de la Escuela de Arquitectura de la Universidad de la Republica del Uruguay en Montevideo (2014). Ambas actividades arrojaron resultados similares.

No es irrelevante comenzar un proceso de proyecto por el estudio del acto (o del usuario) o por el estudio de referentes construidos. No ayuda el hecho de que la literatura sobre el tema sea escasa, de procedencia principalmente chilena y esparcida, siendo Bochers, más un par de cercanos y un grupo de docentes de la UCV, quienes han tratado el problema del acto con cierta profundidad y especificidad.

${ }^{8}$ En tanto se basa en el estudio práctico / lúdico del cuerpo en el espacio principalmente a través de la gimnasia, el Curso Cultura del Cuerpo constituye efectivamente una excepción. 
A continuación, se ofrece un testimonio del

\section{CURSO "FUNDAMENTOS DE LA ARQUITECTURA" COMO ENTORNO ACADÉMICO AD HOC PARA EL ESTUDIO DEL OBJETO DE ESTUDIO PROPIO DEL ARQUITECTO}

Las primeras clases del curso son, de facto. una introducción práctica al paradigma de la fenomenología, a la vez que un viaje en busca del objeto de estudio propio del arquitecto. Concretamente, se llevan a cabo cuatro ejercicios contemplativos sobre el mundo de lo dado, comenzando por una contemplación de un objeto representativo del reino mineral, seguida por uno del reino vegetal, animal y 'humanal', respectivamente. Cada objeto es rodeado en círculo. Durante la primera mitad de cada clase, cada uno, desde su lugar, registra con papel y lápiz, en silencio, lo que ve.

Durante la segunda mitad, se realiza una caracterización exhaustiva de cada objeto, extrayendo un breve manifiesto de cada uno. A continuación, se presentan cuatro diagramas editados de los resultados arrojados por cada clase, compuestos por registros realizados por cada alumno, acompañados a pie de imagen por un breve resumen de cada manifiesto (imágenes 2 ,

\section{3, 4 y 5)}

Esta estrategia didáctica conduce las reflexiones del alumno de forma gradual hacia una idea más o menos clara de cuáles 'no son' objetos de estudio propios del arquitecto. No lo son ni el reino mineral, ni el vegetal ni el animal (sí lo son del geólogo, del botánico y del zoólogo, respectivamente). Sobre el entendido de que la arquitectura no pertenece al mundo de lo dado (natural) sino que al mundo de lo hecho por seres humanos (social), el reino 'humanal' surge como candidato lógico al objeto de estudio propiamente arquitectural. Sin embargo, el sentido común advierte de inmediato que este es el objeto de estudio propio de toda disciplina

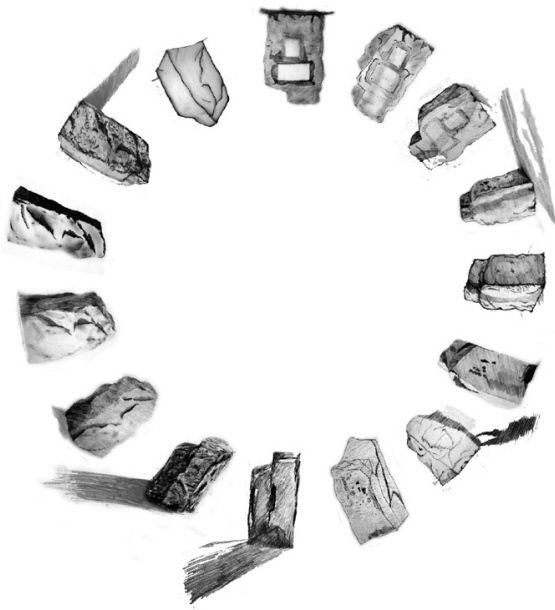

Imagen 2. Caracterización gráfica colectiva de un objeto representativo del reino mineral. El análisis comparado revela a representativo del reino mineral. El análisis comparado revela a
este reino en un estado de 'sueño profundo' (fuente: El autor).

Imagen 3. Caracterización gráfica colectiva de un objeto representativo del reino vegetal. El análisis comparado revela en (fuente: El autor).

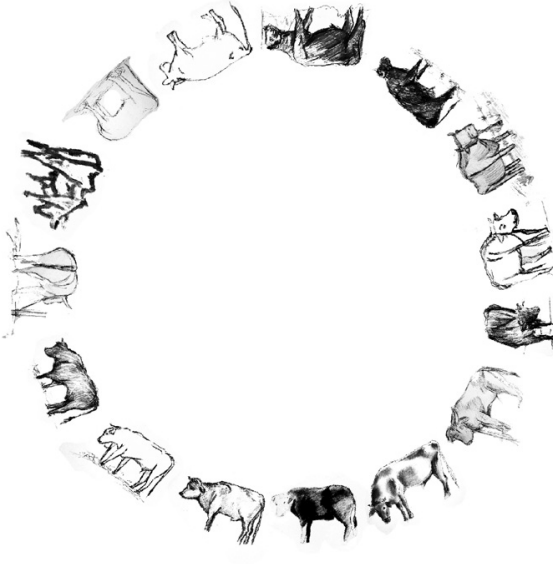

Imagen 4. Caracterización gráfica colectiva de un objeto representativo del reino animal. El análisis comparado revela a este reino como animado, predecible e intrínsecamente completo en su entorno (fuente: El autor). representativo del reino vegetal. El analisis comparado revela en

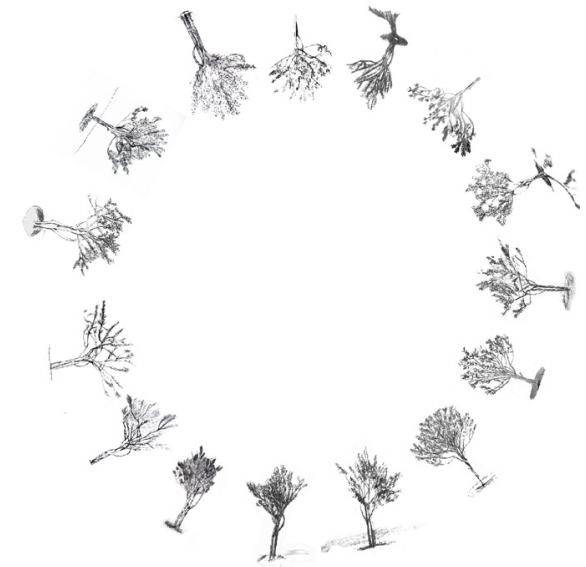

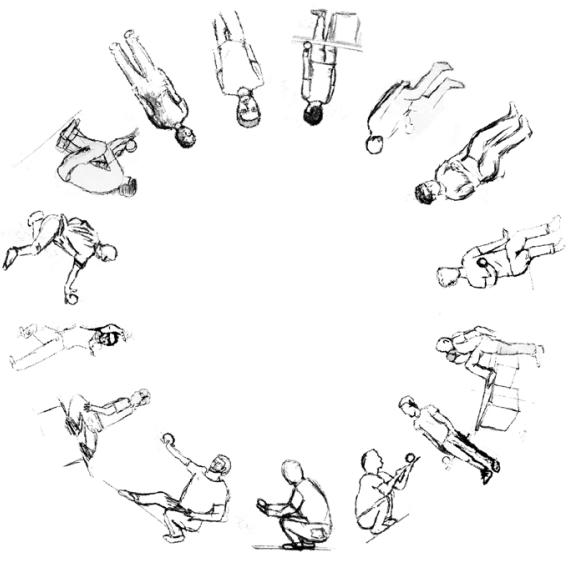

Imagen 5. Caracterización gráfica colectiva de un objeto representativo del reino 'humanal'. El análisis comparado revela a este reino como animado, impredecible e intrinsecamente incompleto en su entorno. Es decir, en necesidad de una morada ad hoc o bien de una tercera piel (fuente: El autor). 
propiamente humana. Surge así la segunda gran pregunta: ¿Qué del reino humanal se constituye como objeto de estudio propio del arquitecto? Esta interrogante da inicio a la segunda parte del curso, la que se basa en la hipótesis de que un principio de respuesta se desprende del estudio de lo que denominamos 'actos humanos arquetípicos', a saber: levantarse/acostarse, caminar/detenerse, sentarse/pararse, encontrarse/desencontrarse, hablar/ escuchar (conversar).

Ceñido disciplinalmente, este comienzo de respuesta a la pregunta central quedaría de la siguiente manera: el estudio del "habitante arquetípico" o bien, del "protofenómeno arquitectónico" (Araneda 2010); ningún habitante o cliente en particular sino el ser humano o naturaleza humana en necesidad de una morada ad hoc (Seamon y Zajonc 1998). Es precisamente la noción goetheana de protofenómeno o fenómeno primordial la que quizás contenga el germen de un nuevo comienzo para los estudios arquitecturales, pues reconecta, de forma actualizada, con la idea de poiesis en el sentido Aristotélico? Aceptando que la detección de este objeto aún no constituye un acto de distinción nítido sino más bien un fenómeno emergente, el desafío práctico consiste en desarrollar estrategias de análisis que se ajusten al objeto en cuestión, pues ocurre que este objeto constituye una suerte de blanco en permanente movimiento. Esto presenta desafíos metodológicos, originalmente detectados y abordados por Eadweard Muybridge en Inglaterra (imagen 6) y por Étienne-Jules Marey en Francia, (imagen 7), ambos pioneros del cine $y$, sin quererlo, de una posible nueva ciencia emergente de los actos humanos o 'actología', la que, llevada hasta sus últimas consecuencias técnicas y tecnológicas, constituye un recurso investigativo de inestimable valor para el estudio del objeto en cuestión.

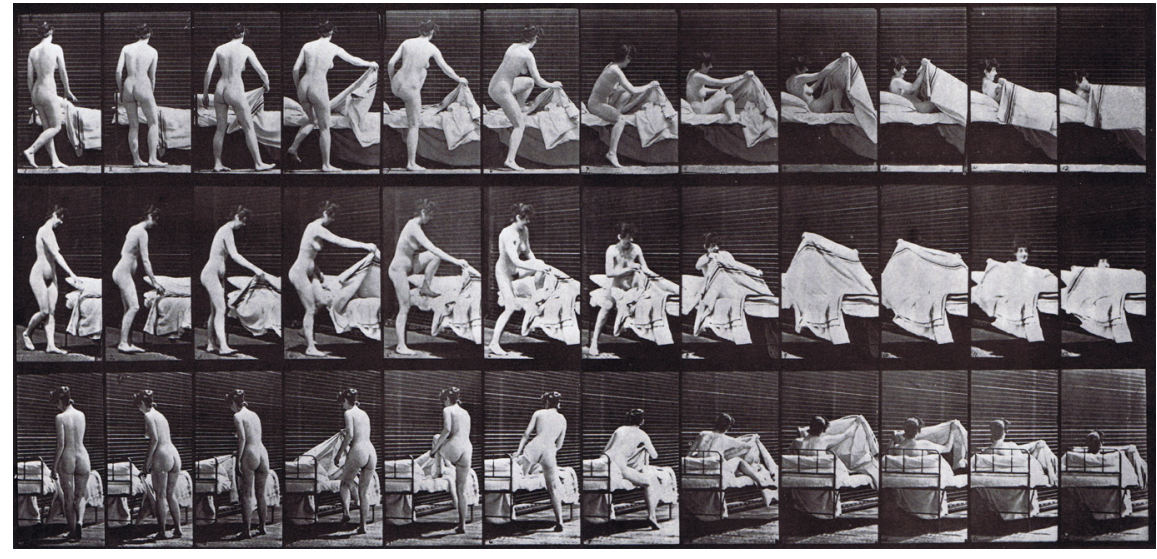

Imagen 6. Eadweard Muybridge (1830-1904). "Getting to bed" (fuente: Adam 2014).

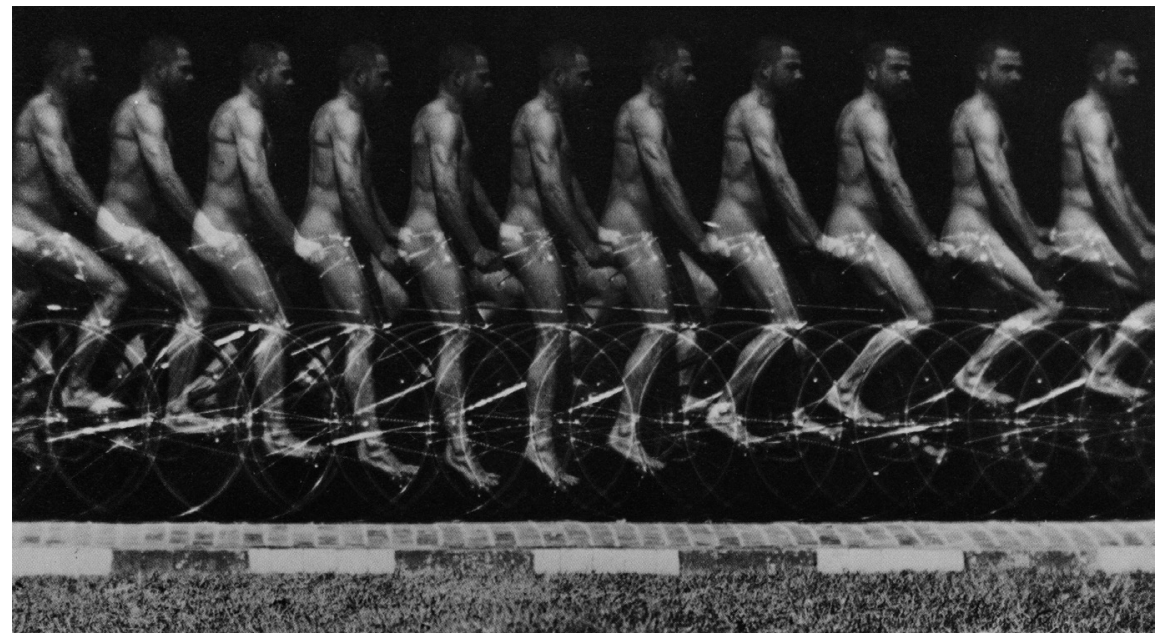

Imagen 7. Etienne-Jules Marey (circa 1891). Hombre desnudo en bicicleta. (Fuente: Frizot 1984). 
CONCLUSIONES. En un contexto académico global caracterizado por el adelgazamiento de contenidos, la apertura de un nicho curricular dedicado al estudio de los fundamentos del fenómeno arquitectónico no puede sino ser considerada como una verdadera conquista académica. En efecto, una revisión somera de las competencias consensuadas y declaradas por los entes responsables de estas reformas en el área, no evidencia claridad respecto a las bases epistemológicas de nuestra disciplina ${ }^{10}$ En este sentido, las reflexiones aqui expuestas sugieren las siguientes hipótesis.
Los hechos construidos no pueden ser nuestro objeto de estudio propio o fuente de conocimiento original. En tanto huellas dejadas por el quehacer del arquitecto, los hechos construidos son más bien objeto de estudio propio del arqueólogo. Dicho de otro modo, con su quehacer, el arquitecto da trabajo al arqueólogo. Para el arquitecto, no obstante, no pueden sino constituir conocimiento de segunda mano. Así visto, el hecho arquitectónico no es fuente $u$ objeto original sino objetivo infinitamente perfectible del arquitecto. De cara al pobre estado del arte en materia epistemológica arquitectónica, la distinción entre objeto y objetivo arquitectónico libera a la vez que empodera. Por un lado, libera al estudiante de arquitectura tanto de la dependencia del profesor como del referente, a la vez que lo empodera como observador crítico y autónomo. Esto, a su vez, otorga deberes y derechos: el deber de meditar de forma sostenida y permanecer fiel a su objeto de estudio (tal como el físico y el biólogo permanecen fieles al universo natural) y, solo como consecuencia de ello, el derecho ganado a la creatividad. En una era caracterizada por la anarquía epistemológica y estética disciplinar, la validez o invalidez de estas hipótesis nos parece digna de ser explorada. $\mathbf{\Delta} \boldsymbol{0}$

\section{REFERENCIAS}

Adam, H., 2014. Muybridge: The Human and Anima/ Locomotion Photographs. China: Taschen. Araneda, C., 2010. "Protofenómeno arquitectónico: Introduciendo la noción de fenómeno primordial en arquitectura" Arquitetura revista, 6 (2): 76-89.

Borchers, J., 1975. Meta-arquitectura. Santiago de Chile: Editorial Andrés Bello. Borchers, J., 1968. Institución Arquitectónica. Santiago de Chile: Editorial Andrés Bello.

Borchers. J. 1965. El Número. Santiago: Fondo Documental Juan Borchers, Archivo de Originales del Centro de Información y Documentación Sergio Larrain García-Moreno, Facultad de Arquitectura, Diseño y Estudios Urbanos, Pontificia Universidad Católica de Chile.

Colomina, B., Eversole, B., Galán, I., Kotsioris, E... Meister, A. y Vannucchi, F., s/f. Radical Pedagogies. Disponible en: http://radical-pedagogies.com/

Cruz, A., 2012. El Acto Arquitectónico. Valparaíso: Ediciones Universitarias de Valparaíso

De la Cruz, R., 2013. "Juan Borchers. El Derecho al Lirismo en Arquitectura." Revista AOA, 23, 28-49

De la Cruz, J., 2000. Alquimia. El acto y e/ número (Tesis doctoral) Pontificia Universidad Católica de Chile, Santiago, Chile

ENHSA, s/f. Publications. Disponible en: http://www.enhsa.net/publications.htm-
Evers, B., 2006. Architectural Theory. Colonia: Taschen.

Frizot, M. 1984. Etienne-Jules Marey. Paris: Centre National de la Photographie. Goethe, J.W. (Von Solar. J. Trad.), 1993. Máximas y Reflexiones, Barcelona: Edhasa Hillier, B., 1996. Space is the Machine. Cambridge: Cambridge University Press. Luhmann, N., 2000. Art as a Social System. California: Stanford University Press.

Pérez, F. Aravena, A. y Quintanilla, J. 1999. Los hechos de la arquitectura. Santiago de Chile: Ediciones ARQ Piñon. H.. 2006. Teoría del Proyecto. Barcelona: Editorial UPC

Proyecto Tuning, 2004-2008. Proyecto Tuning. Disponible en: http://www.unideusto.org/tuning/ Seamon. D. y Zajonc, A.. (Eds.). 1998. Goethe's Way of Science. A Phenomenology of Nature. Nueva York SUNY Press.

Schon, D., 1987. Educating the Reflective Practitioner. San Francisco: Jossey-Bass. Seldin, P., 2010. The Teaching Portfolio. San Francisco: Jossey-Bass.

Van der Laan, H., 1983. Architectonic Space. Leiden, E.J: Bril.

Zubizarreta, J. 2004. The Learning Portfolio. Reflective Practice for Improving Student Learning San

Francisco: Jossey-Bass. 